

\title{
Interplay of Mott Transition and Ferromagnetism in the Orbitally Degenerate Hubbard Model
}

\author{
Raymond Frésard ${ }^{1,2} *$ and Gabriel Kotliar ${ }^{2}$ \\ 1 Physics Department, Shimane University, Nishikawatsu-cho 1060, \\ Matsue 690, Shimane, Japan \\ ${ }^{2}$ Department of Physics and Astronomy, Rutgers University, Piscataway, NJ-08855-0849, USA
}

(October 27, 2018)

\begin{abstract}
A slave boson representation for the degenerate Hubbard model is introduced. The location of the metal to insulator transition that occurs at commensurate densities is shown to depend weakly on the band degeneracy $M$. The relative weights of the Hubbard sub-bands depend strongly on $M$, as well as the magnetic properties. It is also shown that a sizable Hund's rule coupling is required in order to have a ferromagnetic instability appearing. The metal to insulator transition driven by an increase in temperature is a strong function of it.
\end{abstract}

PACS number: 71.27.+a, 71.28.+d, 71.30.+h, 72,80.Ga

There has been dramatic progress in our understanding of the Mott transition in the last few years. Careful experimental studies of systems in the vicinity of the Mott transition have been carried out and two new theoretical fools, slave bosons mean field theories (see for instance $\mathrm{B}$ and references therein), and the limit of infinite dimensions have been adapted to its study. For a review sed3. Most of the modern work has focused on the single band Hubbard model. Now that both the metallic and the Mott insulating states of the (doped) titanates and vanadiates have been studied experimentally 4 (corresponding to $3 d 1$ and $3 d 2$ configurations in the Mott insulating state), there is a need for a theoretical framework allowing for understanding the Mott transition for arbitrary degeneracy and density. This paper is aimed to provide such a technique and to apply it to a variety of quantities that cannot be obtained easily using alternative approaches. Most of the results are obtained in a closed analytical form, allowing for a qualitative understanding of the physical situation.

In this work we investigate the effect of strong Coulomb interaction in systems with orbital degeneracy. Such a situation is realized in virtually all transition metals and transition metal oxides. These systems contain d electrons in cubic or trigonal environments, the crystal field can only lift partially the degeneracy of the d-bands, down to 2 as is the case of $\mathrm{V}_{2} \mathrm{O}_{3}$ ] or 3 as in $\mathrm{LaTiO}_{3}$. Our goal is to understand how degeneracy affects the behavior of the different physical quantities near the Mott transition. To carry out the investigation we extend the slave boson technique which has been very successful in the study of the Mott transition, to the orbitally degenerate case. Compared to the variational wave function approach demonstrate in this paper, it allows us to calculate a variety of quantities which are not easily accessible to the variational approach, as a function of the correlation strength and doping. It can also be improved systematically by performing a loop expansion around the saddle point. Our main results are the following :a) low energy single particle quantities such as the critical value of the interaction strength at which the transition occurs, the quasiparticle residue and the single particle Mott Hubbard gap depend very weekly on degeneracy justifying the agreement between theory and experiment when it was applied to orbitally degenerate systems. b) the relative weights of the Hubbard bands depend strongly on degeneracy in agreement with other methods.6 c) the degeneracy temperature decreases with increasing band degeneracy. d) the magnetic properties, the magnetic susceptibility and its associated Landau parameter in the paramagnetic phase and the magnetic phase diagram is strongly modified from the one band case.

The Hamiltonian describing the low-energy properties of these systems is commonly written as:

$$
\begin{aligned}
H & =\sum_{i, j, \sigma, \rho} t_{i, j} c_{i, \rho, \sigma}^{+} c_{j, \rho, \sigma}+U_{3} \sum_{i, \rho} n_{i, \rho, \uparrow} n_{i, \rho, \downarrow} \\
& +U_{1} \sum_{i, \rho^{\prime} \neq \rho} n_{i, \rho, \uparrow} n_{i, \rho^{\prime}, \downarrow}+U \sum_{i, \sigma, \rho^{\prime}<\rho} n_{i, \rho, \sigma} n_{i, \rho^{\prime}, \sigma}
\end{aligned}
$$

where $\sigma$ is a spin index for the up and down states while $\rho$ is labeling the $\mathrm{M}$ bands, and $U_{n} \equiv U+n J$. Taking $J$ finite accounts for the Hund's rule coupling.

As for any model with on-site interaction, a slave boson representation can be introduced, mapping all the degrees of freedom onto bosons. We can re-write any atomic

*Present address: Institut de Physique, Université de Neuchâtel, A.-L. Breguet 1, CH-2000 Neuchâtel 
state with the help of a set of pseudo-fermions $\left\{f_{\alpha}\right\}$ and slave bosons $\left\{\psi_{\alpha_{1}, \ldots \alpha_{m}}^{(m)}\right\}(0 \leq m \leq 2 M) . \psi_{\alpha_{1}, \ldots \alpha_{m}}^{(m)}$ is the slave boson associated with the atomic state consisting of $m$ electrons in states $\left|\alpha_{1}, \ldots, \alpha_{m}\right\rangle$ where $\alpha$ is a composite spin and band index. By construction it is symmetric under any permutation of 2 indices, and 0 if any 2 indices are equal. We can now write the creation operator of a physical electron in terms of the slave particles as:

$$
c_{\alpha}^{+}=\tilde{z}_{\alpha}^{+} f_{\alpha}^{+}
$$

$\tilde{z}_{\alpha}^{+}$describes the change in the boson occupation numbers when an electron in state $\alpha$ is created as:

$$
\tilde{z}_{\alpha}^{+}=\sum_{m=1}^{2 M} \sum_{\alpha_{1}<.<\alpha_{m-1}} \psi_{\alpha, \alpha_{1}, \ldots, \alpha_{m-1}}^{+(m)} \psi_{\alpha_{1}, \ldots, \alpha_{m-1}}^{(m-1)} \quad \alpha_{i} \neq \alpha
$$

The operators $\tilde{z}_{\alpha}^{+}$in Eq. (3) describes the change in the slave boson occupation as a many channel process. In order to recover the correct non-interacting limit at mean-field level, one has to observe that the classical probability for these processes to happen is not simply given by taking the Bose fields in (3) to be given by their classical values, but by introducing normalization factors $L_{\alpha}$ and $R_{\alpha}$ e. as $z_{\alpha}^{+}=\sum \psi^{+(m)} L_{\alpha} R_{\alpha} \psi^{(m-1)}$, where:

$$
\begin{aligned}
R_{\alpha} & =\left[1-\sum_{m=0}^{2 M-1} \sum_{\alpha_{1},<.<, \alpha_{m}} \psi_{\alpha_{1}, ., \alpha_{m}}^{+(m)} \psi_{\alpha_{1}, ., \alpha_{m}}^{(m)}\right]^{-\frac{1}{2}} \alpha_{i} \neq \alpha \\
L_{\alpha} & =\left[1-\sum_{m=1}^{2 M} \sum_{\alpha_{1}<.<\alpha_{m-1}} \psi_{\alpha, \alpha_{1}, ., \alpha_{m-1}}^{+(m)} \psi_{\alpha, \alpha_{1}, ., \alpha_{m-1}}^{(m)}\right]^{-\frac{1}{2}} .
\end{aligned}
$$

Namely $L_{\alpha}$ normalizes to 1 the probability that no electron in state $|\alpha\rangle$ is present on a site before one such electron hops to that particular site, and $R_{\alpha}$ makes sure that it happened. Clearly the eigenvalues of the operators $L_{\alpha}$ and $R_{\alpha}$ are 1 in the physical subspace. Now, the redundant degrees of freedom are projected out with the constraints:

$$
\begin{gathered}
f_{\alpha}^{+} f_{\alpha}-\sum_{m=1}^{2 M} \sum_{\alpha_{1}<.<\alpha_{m-1}} \psi_{\alpha, \alpha_{1}, ., \alpha_{m-1}}^{+(m)} \psi_{\alpha, \alpha_{1}, ., \alpha_{m-1}}^{(m)}=0 \\
\sum_{m=0}^{2 M} \sum_{\alpha_{1}<.<\alpha_{m}} \psi_{\alpha_{1}, ., \alpha_{m}}^{+(m)} \psi_{\alpha_{1}, ., \alpha_{m}}^{(m)}-1=0
\end{gathered}
$$

We obtain the Lagrangian at $J=0$ as:

$$
\begin{aligned}
& L=\sum_{i \alpha} f_{i, \alpha}^{+}\left(\partial_{\tau}-\mu+i \lambda_{i, \alpha}\right) f_{i, \alpha}-i \Lambda_{i}+\sum_{i, m} \sum_{i \alpha_{1}<,<\alpha_{m}} \\
& \psi_{i, \alpha_{1}, ., \alpha_{m}}^{+(m)}\left(\partial_{\tau}+i \Lambda_{i}+U\left(\begin{array}{c}
m \\
2
\end{array}\right)-i \sum_{j=1}^{m} \lambda_{i, \alpha_{j}}\right) \psi_{i, \alpha_{1}, ., \alpha_{m}}^{(m)} \\
& +\sum_{i, j, \alpha} t_{i, j} z_{i, \alpha}^{+} f_{i, \alpha}^{+} z_{j, \alpha} f_{j, m, \alpha} .
\end{aligned}
$$

We now proceed to the mean-field theory, and we investigate the paramagnetic, paraorbital saddle-point. The latter is obtained after integrating out the fermions, and setting all bosonic fields to their classical value. The Mott transition that occurs at commensurate density $n$ is best discussed by projecting out occupancies that are larger than $n+1$ and smaller than $n-1$ (if any). The constraints allows for eliminating the variables $\psi^{(n-1)}$ and $\psi^{(n)}$ to obtain the grand potential at $n$ :

$$
\begin{aligned}
\Omega(D) & =\left(1-2 D^{2}\right) D^{2}\left(\sqrt{b_{n, M}}+\sqrt{c_{n}}\right)^{2} \epsilon_{0} \\
& +U\left(D^{2}+\left(\begin{array}{l}
n \\
2
\end{array}\right)\right)-\mu \rho
\end{aligned}
$$

with $\epsilon_{0} \equiv 2 M \int d \epsilon \epsilon \rho(\epsilon) f_{F}\left(z^{2} \epsilon+\lambda_{0}-\mu\right), \quad D^{2} \equiv$ $\left(\begin{array}{c}2 M \\ n+1\end{array}\right) \psi^{(n+1) 2}, b_{n, M} \equiv(2 M-n+1) /(2 M-n)$, and $c_{n} \equiv(n+1) / n$. Minimizing Eq. (7) with respect to $D$ yields a critical interaction strength at which $D$ vanishes. It reads $U_{c}^{(n, M)}=-\epsilon_{0}\left(\sqrt{b_{n, M}}+\sqrt{c_{n}}\right)^{2}$ which reproduces the results of the Gutzwiller approximation 78 . This locates the Mott transition. Restricting ourselves to a flat density of states we can relate the critical interaction strength to the band width $W$. We obtain:

$$
U_{c}^{(n, M)}=\frac{n W}{4 M}(2 M-n)\left(\sqrt{b_{n, M}}+\sqrt{c_{n}}\right)^{2}
$$

Its band degeneracy dependence is fairly weak $\mathrm{A}$. The effective mass of the quasi-particles diverges at the Mott transition. We obtain:

$$
\frac{m}{m^{*}}=z^{2}=\frac{\left(\sqrt{b_{n, M}}+\sqrt{c_{n}}\right)^{2}}{8} \quad \frac{U_{c}^{(n, M) 2}-U^{2}}{U_{c}^{(n, M) 2}}
$$

Due to the particular form of the coefficients $b$ and $c$ the dependence on the band degeneracy is weak. The critical interaction strength increases with $M$ so the quasiparticle residue $Z$ increases slightly with $\mathrm{M}$. For small values of $\mathrm{U}$, (which we treated without projecting out higher occupancies), $\mathrm{Z}$ decreases with increasing $M$. So there is a crossover value of the interaction strength beyond which the system becomes more metallic with increasing $M$. As a function of the hole doping $\delta$, the quasi-particle residue vanishes for $\delta$ going to 0 above $U_{c}^{(n, M)}$ as:

$$
\begin{aligned}
z^{2} & =\frac{\delta}{2}\left(b_{n, M}-c_{n}\right)+\frac{|\delta|}{2}\left(\left(b_{n, M}+c_{n}\right)\right. \\
& \left.\times \sqrt{1+4 \varphi_{n, M}}+4 \sqrt{b_{n, M} c_{n} \varphi_{n, M}}\right)
\end{aligned}
$$

where we introduced:

$$
\varphi_{n, M} \equiv \frac{U_{c}^{(n, M) 2} \frac{b_{n, M} c_{n}}{\left(\sqrt{b_{n, M}}+\sqrt{c_{n}}\right)^{4}}}{\left(U-U_{c}^{(n, M)}\right)\left(U-U_{c}^{(n, M)}\left(\frac{\sqrt{b_{n, M}}-\sqrt{c_{n}}}{\sqrt{b_{n, M}}+\sqrt{c_{n}}}\right)^{2}\right)}
$$

The expression of the quasi-particle residue consists of 2 contributions which are either symmetric or antisymmetric with respect to particle or hole doping. The antisymmetric contribution vanishes for $n=M$ as a consequence 
of the particle-hole symmetry. The asymmetry of $z^{2}$ on particle or hole doping is seen to increase under an increase of $|n-M|$. It vanishes more slowly for hole doping (for $n \leq M$ ) than for particle doping, for increasing degeneracy at fixed $n$, for increasing degeneracy at $n=M$ and under an increase of $U$. As an example we calculate the effective mass for the 2 band model and show it on Fig. 1, which has been calculated without projecting out higher occupancies.

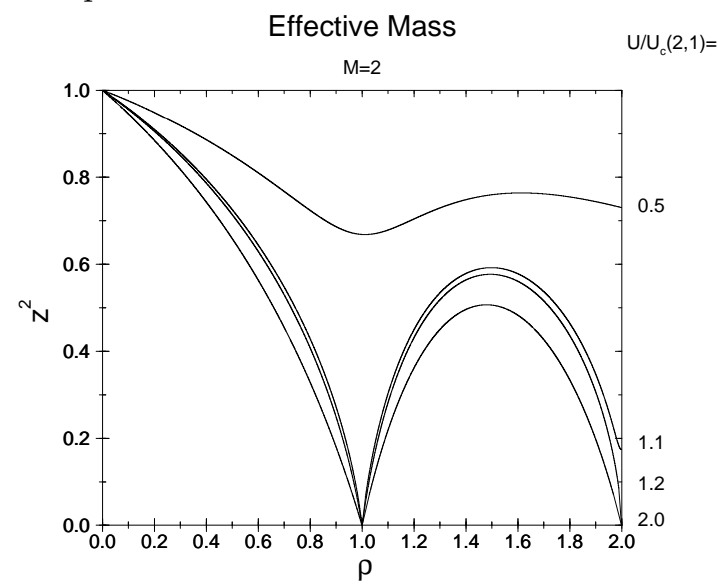

FIG. 1. Inverse effective mass in the 2 band model as a function of density for several values of $U$.

Interestingly we also obtain a Mott gap. Indeed the number of quasi-particles is a continuous function of their chemical potential $\mu-\lambda_{0} / 2$. However the saddle-point equations show that the Lagrange multiplier $\Lambda$ jumps when going through the Mott gap which implies that $\lambda$ is going to jump as well, and so does $\mu$. As a result we obtain the Mott gap $\Delta \equiv \lim _{\delta \rightarrow 0^{-}} \mu(\delta)-\lim _{\delta \rightarrow 0^{+}} \mu(\delta)$ as:

$$
\Delta=\sqrt{\left(U-U_{c}^{(n, M)}\right)\left(U-U_{c}^{(n, M)}\left(\frac{\sqrt{b_{n, M}}-\sqrt{c_{n}}}{\sqrt{b_{n, M}}+\sqrt{c_{n}}}\right)^{2}\right)}
$$

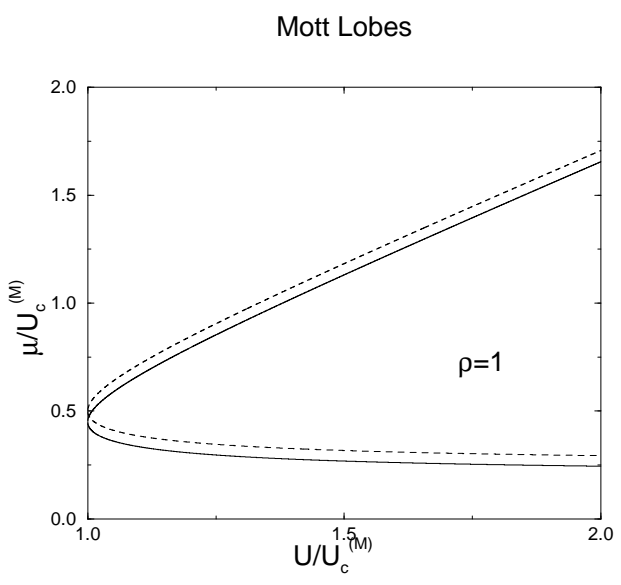

FIG. 2. Chemical potential for $n=1$ for the 1 band (dashed line) and 2 band (full line) models.

In the limit of $U>U_{c}^{(n, M)}$, the Mott gap is given by $U$, while it closes at $U_{c}^{(n, M)}$ as $\Delta \sim$ $U_{c}^{(n, M)} \sqrt{U / U_{c}^{(n, M)}-1}$, the square root behavior being typical of slave boson mean-field theories. It can be read from Fig. 2 where it is compared to the 1 band case as obtained by Lavagna 11 . Clearly going from 1 band to 2 bands does not imply a big difference in the Mott gap. Indeed we obtain the $\Delta / U_{c}^{(n, M)}$ is independent of $M$ at $n=M$, while for fixed $n$ it depends very weakly on $M$.

Our result can be compared to experimental data. For the series $\mathrm{La}_{x} Y_{1-x} \mathrm{TiO}_{3}$, Okimoto et a 12 measured how the gap depends on the band width. Assuming (for large ratio $U / W) \Delta \sim U-W$ we obtain out of their data $U=3.2 \mathrm{eV}$. Inserting this and the experimental value of $(U / W)_{c} \sim 1.3$ in Eq.(12) we can compute $\Delta / W$ as a function of $W / U$ and compare it with experiment on fig. 3. The experimental trend is clearly reproduced and the quantitative agreement is very satisfactory.

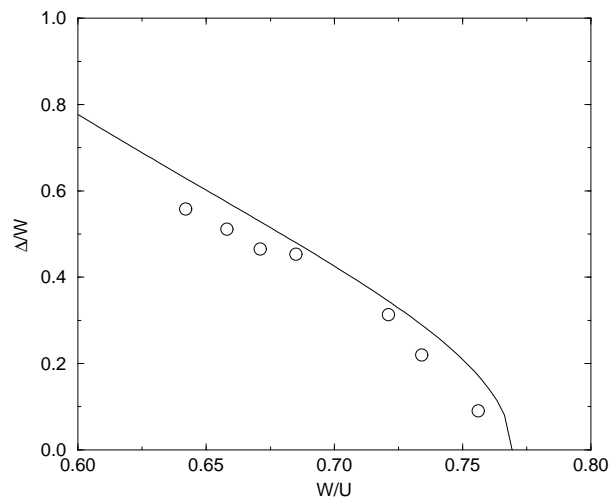

FIG. 3. Dependence of the Mott gap on the band width for $n=1$ and $M=3$. Circles: experimental data of Okimoto et $a l^{12}$.

We now turn to the Hund's rule coupling dependence and treat as an example the two band model around the $n=1$ Mott insulating lobe. At $\rho=1$ the grand potential at the saddle-point reads:

$$
\begin{aligned}
\Omega & =\frac{4}{3} \epsilon_{0}\left(1-2 r^{2}\right)\left(r+\left(d_{0}+d_{x}+\Delta_{0}\right) / \sqrt{2}\right)^{2} \\
& +(U+3 J) \Delta_{0}^{2}+(U+J) d_{x}^{2}+U d_{0}^{2}-\mu \rho .
\end{aligned}
$$

with $d_{0} \equiv\left(\psi_{\uparrow, \uparrow}^{(2)}+\psi_{\downarrow, \downarrow}^{(2)}\right) / \sqrt{2}, d_{x} \equiv\left(\psi_{\uparrow, \downarrow}^{(2)}+\psi_{\downarrow, \uparrow}^{(2)}\right) / \sqrt{2}$, $\Delta_{0} \equiv\left(\psi_{\uparrow \downarrow, 0}^{(2)}+\psi_{0, \downarrow \uparrow}^{(2)}\right) / \sqrt{2}, r^{2} \equiv d_{0}^{2}+d_{x}^{2}+\Delta_{0}^{2}$ and $\lambda \equiv \sum_{\alpha} \lambda_{\alpha} / 2$, and we have used the constraints to remove the variables $\psi^{(0)}$ and $\psi^{(1)}$.

Such an expression differs from an ordinary GinzburgLandau free energy in that respect that it cannot be written as a 4 th order polynomial in the variables $d_{0}, d_{x}$ and 
$\Delta_{0}$. As a result, if there were to be a critical point for 1 field, it would be critical for the other ones as well. We obtain the location of the Mott transition as:

$$
U_{c,(J)}^{(2)}=U_{c,(0)}^{(2)}\left(1-\frac{4}{3} \quad \frac{J}{U}+O(J / U)^{2}\right) \quad .
$$

Another regime of interest is the large $J$ regime. There we obtain the location of the Mott transition as:

$$
U_{c}^{(2)}=-\frac{2}{3} \epsilon_{0}(3+2 \sqrt{2})\left(1-\frac{8}{9} \frac{\epsilon_{0}}{J}\right)+O\left(\left(\frac{\epsilon_{0}}{J}\right)^{2}\right)
$$

and thus decreasing $J$ from $\infty$ leads to an increase of the critical interaction. Another intriguing feature of transition metal oxides such as $\mathrm{V}_{2} \mathrm{O}_{3}$, is the metal to insulator transition that occurs in the vicinity of the tri-critical point under an increase of temperature. It has recently been interpretated 5 as the transition from a Fermi liquid with finite quasi-particle residue $Z$ to an insulator with $Z=0$. In other words there is a finite coherence temperature $T_{c o h}$ at which the coherence of the Fermi liquid (and $Z$ ) vanishes. This result was obtained in the dynamical mean-field approximation to the 1 band model, which becomes exact in the limit of large dimensions, and recovered in the Gutzwiller Approximation 14. At finite $T$ there is a first order metal to insulator transition at a critical $U_{c}^{(M)}(T)$ :

$$
U_{c}^{(M)}(T)=U_{c}^{(M)}(0)-\sqrt{8 U_{c}^{(M)}(0) T \ln 2 M}
$$

Thus an increase in temperature may produce a metal to insulator transition, which is consistent with the experimental situation in $\mathrm{V}_{2} \mathrm{O}_{3}$. In the dynamical mean-field approximation at finite temperatures there is an interaction strength $U_{c 2}(T)$ at which the metallic solution ceases to exist. This quantity can also be evaluated in our slave boson scheme and is given by:

$$
U_{c 2}^{(M)}(T)=U_{c}^{(M)}(0)\left(1-\alpha_{M}(T / W)^{\frac{2}{3}}\right)
$$

with $\alpha_{1} \sim 2.53$ and $\alpha_{2} \sim 3.32$.

We now turn to the calculation of the magnetic susceptibility. Here we generalize the calculation of $\mathrm{Li}$ et $a, 13$ to the 2 band model. The linear response to an external magnetic field is obtained as a 1 loop calculation of the correlation function of the slave boson fields in the spin-antisymmetric band-symmetric channel. 3 fields couple in this channel10: $\chi_{-} \equiv \frac{1}{2} \sum_{\alpha} \sigma \psi_{\alpha}^{(1)}$, $\chi_{+} \equiv \frac{1}{\sqrt{2}}\left(\psi_{\uparrow, \uparrow}^{(2)}-\psi_{\downarrow, \downarrow}^{(2)}\right)$ and $\kappa \equiv \frac{1}{2} \sum_{\alpha} \sigma \lambda_{\alpha}$, and the magnetization is expressed in terms of slave bosons as $M=4 d_{0} \chi_{+}+2 \psi_{i}^{(1)} \chi_{-}$. The resulting susceptibility arises as an RPA form 10

$$
\chi_{S}=\frac{\chi_{0}}{1+F_{0}^{a} \chi_{0} / N\left(E_{F}\right)} .
$$

We now determine the instability line of the paramagnetic phase with respect to ferromagnetism. For $J=0$ we find no ferromagnetic instability even near the Mott transition, while for finite $J$ we find that the Mott metal insulator transition may be preempted by the appearance of a ferromagnetic phase. In other words, a sufficiently strong Hund's rule coupling turns a Mott insulator into a ferromagnet. Originally the Hubbard model was introduced in order to describe ferromagnetism in narrow band systems, but it has been recently established that the ground state is not ferromagnetic for any reasonable values of the parameters on generic lattices 16.17 . We find that the ground state is much more likely to be ferromagnetic in the degenerate model for finite $J$, as shown in Fig. 田.

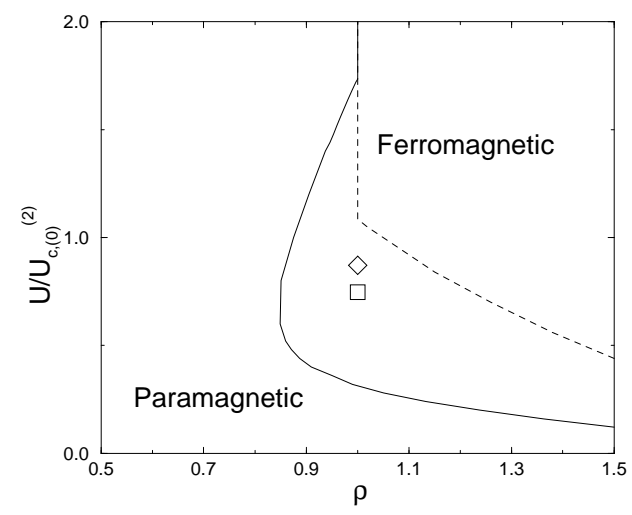

FIG. 4. Instability line of the paramagnetic phase for $U / J=10$ (dashed line) and $U / J=5$ (solid line). The diamond (square) indicates the position of the Mott transition for $U / J=10(U / J=5)$.

Our method can be applied to the calculation of dynamical quantities too. In the strong coupling regime the one-particle excitation spectrum is split off into several pieces, each of them carrying some fraction of the spectral weight (which are adding up to 1 so as to fulfill the sum rule). The various pieces are following from the discrete atomic levels, which are well separated by multiples of $U$, broadened by exchange processes. Let us now determine the fraction of the spectral weight carried by each sub-band. In our language the low energy excitations are involving the field $\psi^{(1)}$, and the high energy excitations centered around $U$ the field $\psi^{(2)}$. Higher energy excitations involving higher local occupancies are left out. We obtain the spectral weights in both bands from the decomposition of the physical electron operator (Eqs. (2,3)). Accordingly the spectral weight of the Green's function $T<c_{\alpha}(\tau) c_{\alpha}^{+}(0)>$ in the lower Hubbard band $\left(W_{L H B}\right)$ and the upper Hubbard band $\left(W_{U H B}\right)$ are given by:

$$
\begin{aligned}
W_{L H B} & =<\psi^{+(0)} \psi^{(0)}+\psi_{\alpha}^{+(1)} \psi_{\alpha}^{(1)}> \\
W_{U H B} & =\sum_{\beta \neq \alpha}<\psi_{\beta}^{+(1)} \psi_{\beta}^{(1)}+\psi_{\alpha \beta}^{+(2)} \psi_{\alpha \beta}^{(2)}>
\end{aligned}
$$

and are shown in fig. 5. Here the weights do not quite 
add up to 1 in the 2 band case because we projected out occupations larger than 2. In other words, on top of the 2 sub-bands which are considered here, there appear a second upper Hubbard band (centered around $3 U-3 \mu$, corresponding to triple occupancy) which is becoming relevant in the particle doped regime. To a very good accuracy its contribution to the spectral weight is given by $1-W_{L H B}-W_{U H B}$. Clearly the degeneracy plays an important role as the weight of the upper band at $n=1$ in the strong coupling regime is given by $(2 M-1) / 2 M$.

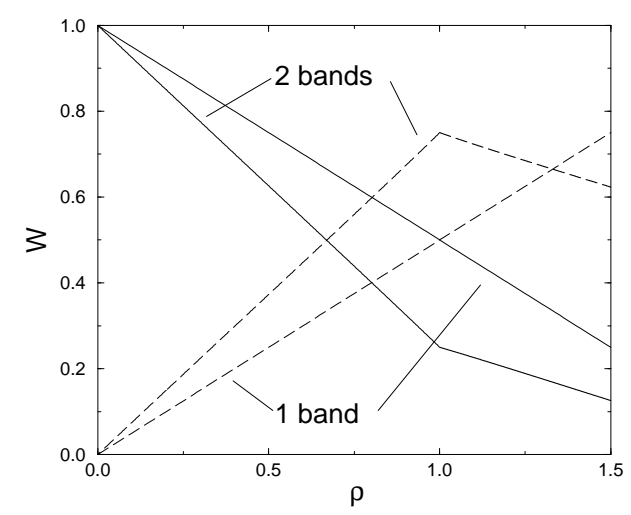

FIG. 5. Spectral weight of the upper (dashed lines) and lower (solid lines) Hubbard bands for the 1 band and 2 band models, at $U=2 U_{c}^{(M)}$.

In summary we introduced a slave boson representation of the degenerate Hubbard model. We obtained that the band degeneracy has a weak influence on the location of the Mott transition, while the degeneracy temperature and the dynamical and magnetic properties strongly depend on it. We also showed that no ferromagnetic instability occurs unless the Hund's rule coupling becomes sizable, yielding a generic scenario for ferromagnetism in transition metals and transition metal oxides. In that case a ferromagnetic instability may even shadow the
Mott transition. RF gratefully acknowledges financial support from the Fonds National Suisse de la Recherche Scientifique, as well as Rutgers University and Neuchâtel University for hospitality were part of this work has been done. This work has been partially supported by the NSF under grant DMR 95-29138.

${ }^{1}$ Y. Tokura et al, Phys. Rev. Lett. 70, 2126 (1993).

${ }^{2}$ R. Frésard and P. Wölfle, Proc. of the Adriatico Research Conference and Miniworkshop Strongly Correlated Electron Systems III, Eds. G. Baskaran et al, Int. J. of Mod. Phys. B 6, 685 (1992); Erratum, Int. J. Mod. Phys. B 6, 3087 (1992).

${ }^{3}$ A. Georges et al, Rev. Mod. Phys. 68, 13 (1996).

${ }^{4}$ H.C. Nguyen and J.B. Goodenough, Phys. Rev. B 52, 8776 (1995); F. Inaba et al, Phys. Rev. B 52, R2221 (1995)

${ }^{5}$ C. Castellani et al, Phys. Rev. B 18, 4945 (1978).

${ }^{6}$ H. Eskes et al, Phys. Rev. B 50, 17980 (1994).

${ }^{7}$ K.A. Chao and M. Gutzwiller, J. Appl. Phys. 42, 1420 (1971). K.A. Chao J. Pys. C 7, 127 (1974); J. Bünemann and W. Weber, Preprint Sissa server cond-mat/9611032.

8 J. P. Lu, Phys. Reb. B 49, 5687 (1994).

${ }^{9}$ G. Kotliar and A. E. Ruckenstein, Phys. Rev. Lett. 57, 1362 (1986).

${ }^{10}$ R. Frésard, G. Kotliar (unpublished)

${ }^{11}$ M. Lavagna, Phys. Rev. B 41, 142 (1990)

${ }^{12}$ Y. Okimoto et al, Phys. Rev. B 51, 9581 (1995).

13 T. Li, Y.S. Sun and P. Wölfle, Z. Phys. B 82, 369 (1991).

${ }^{14}$ R. Frésard and K. Doll, Proc. of the NATO ARW "The Hubbard Model: Its Physics and Mathematical Physics", Ed. D. Baeriswil et al, San Sebastian (1993), Plenum (1995), p.385

15 M. J. Rozenberg et al, Phys. Rev. Lett. 75, 105 (1995)

${ }^{16}$ B. Möller et al, J. Phys.: Cond. Matt. 5, 4847 (1993).

${ }^{17} \mathrm{P}$. Wurth et al Sissa Preprint cond-mat/9512060. 